\title{
Constructions of Bipartite and Bipartite-regular Hypermaps
}

\author{
Rui Duarte \\ Department of Mathematics \\ University of Aveiro \\ 3810-193 Aveiro, Portugal \\ rduarte@ua.pt
}

Submitted: Sep 21, 2011; Accepted: Jun 4, 2012; Published: Oct 18, 2012

Mathematics Subject Classifications: 05C10, 05C25, 20B25

\begin{abstract}
A hypermap is bipartite if its set of flags can be divided into two parts $A$ and $B$ so that both $A$ and $B$ are the union of vertices, and consecutive vertices around an edge or a face are contained in alternate parts. A bipartite hypermap is bipartiteregular if its set of automorphisms is transitive on $A$ and on $B$.

In this paper we see some properties of the constructions of bipartite hypermaps described algebraically by Breda and Duarte in 2007 which generalize the construction induced by the Walsh representation of hypermaps. As an application we show that all surfaces have bipartite-regular hypermaps.
\end{abstract}

Keywords: Hypermap, bipartite hypermap, operations on hypermaps

\section{Definitions and notation}

A hypermap $\mathcal{H}$ is a 4 -tuple $\left(|\mathcal{H}|, h_{0}, h_{1}, h_{2}\right)$, where $|\mathcal{H}|$ is a non-empty finite set and $h_{0}$, $h_{1}$ and $h_{2}$ are involutory permutations on $|\mathcal{H}|$ generating a transitive group $\operatorname{Mon}(\mathcal{H})$, the monodromy group of $\mathcal{H}$. The elements of $|\mathcal{H}|$ are called flags and the orbits of the dihedral subgroups $\left\langle h_{1}, h_{2}\right\rangle,\left\langle h_{2}, h_{0}\right\rangle$ and $\left\langle h_{0}, h_{1}\right\rangle$ are called hypervertices, hyperedges and hyperfaces of $\mathcal{H}$. We use vertices, edges and faces instead of hypervertices, hyperedges and hyperfaces, for short. When $h_{2} h_{0}$ has order $2, \mathcal{H}$ is a map. Topologically, a hypermap resp. map is a cellular embedding of a connected hypergraph resp. graph into a closed connected surface, usually called the underlying surface of the hypermap resp. map.

Given $\mathcal{G}=\left(|\mathcal{G}|, g_{0}, g_{1}, g_{2}\right)$ and $\mathcal{H}=\left(|\mathcal{H}|, h_{0}, h_{1}, h_{2}\right)$ hypermaps, a covering from $\mathcal{G}$ to $\mathcal{H}$ is a function $\psi:|\mathcal{G}| \rightarrow|\mathcal{H}|$ such that $g_{i} \psi=\psi h_{i}$ for all $i \in\{0,1,2\}$. When $\psi$ is one-to-one, $\psi$ is called an isomorphism. If there is a covering $\psi$ from $\mathcal{G}$ to $\mathcal{H}$, we say that $\mathcal{G}$ covers $\mathcal{H}$ or that $\mathcal{H}$ is covered by $\mathcal{G}$, and write $\mathcal{G} \rightarrow \mathcal{H}$; if $\psi$ is an isomorphism, we say that $\mathcal{G}$ 
is isomorphic to $\mathcal{H}$ or that $\mathcal{G}$ and $\mathcal{H}$ are isomorphic and write $\mathcal{G} \cong \mathcal{H}$. An automorphism or symmetry of $\mathcal{H}$ is an isomorphism from $\mathcal{H}$ to itself. The set of automorphisms of $\mathcal{H}$ is denoted by $\operatorname{Aut}(\mathcal{H})$.

For any hypermap $\mathcal{H}$ we have a transitive permutation representation $\Delta \rightarrow \operatorname{Mon}(\mathcal{H})$ of the free product

$$
\Delta=\left\langle R_{0}, R_{1}, R_{2} \mid R_{0}^{2}=R_{1}^{2}=R_{2}^{2}=1\right\rangle \cong C_{2} * C_{2} * C_{2}
$$

The stabilizer $H$ of a flag under the action of $\Delta$, unique up to conjugacy, is called a hypermap subgroup for $\mathcal{H}$. It is well known that hypermap subgroups completely describe hypermaps.

Let $\Theta$ be a normal subgroup of $\Delta$. Following [1], $\mathcal{H}$ is said to be $\Theta$-conservative if $H \leqslant \Theta$, and $\Theta$-regular if $H$ is normal in $\Theta$. A $\Delta$-regular hypermap is best known as regular. The even word subgroup of $\Delta$ is the subgroup

$$
\Delta^{+}=\left\langle R_{1} R_{2}, R_{2} R_{0}, R_{0} R_{1}\right\rangle
$$

which is one of the seven normal subgroups of index 2 in $\Delta$ [3]. The others are

$$
\begin{array}{lll}
\Delta^{\hat{0}}=\left\langle R_{1}, R_{2}\right\rangle^{\Delta}, & \Delta^{\hat{1}}=\left\langle R_{2}, R_{0}\right\rangle^{\Delta}, & \Delta^{\hat{2}}=\left\langle R_{0}, R_{1}\right\rangle^{\Delta}, \\
\Delta^{0}=\left\langle R_{0}, R_{1} R_{2}\right\rangle^{\Delta}, & \Delta^{1}=\left\langle R_{1}, R_{2} R_{0}\right\rangle^{\Delta}, & \Delta^{2}=\left\langle R_{2}, R_{0} R_{1}\right\rangle^{\Delta} .
\end{array}
$$

Usually $\Delta^{+}$-conservative and $\Delta^{+}$-regular hypermaps are called orientable and orientablyregular, respectively. An orientably-regular hypermap which is not regular is called chiral. Following [1,2], a hypermap is bipartite if it is $\Delta^{0}$-conservative, and bipartite-regular if it is $\Delta^{\hat{0}}$-regular. We say that $\mathcal{H}$ has no boundary if no conjugate of $R_{0}, R_{1}$ or $R_{2}$ in $\Delta$ belongs to $H$. Because $R_{0} \notin \Delta^{\hat{0}}$, a bipartite hypermap has no boundary if and only if no conjugate of $R_{1}, R_{2}, R_{1}{ }^{R_{0}}$ or $R_{2}{ }^{R_{0}}$ in $\Delta^{\hat{0}}$ belongs to $H$. A hypermap which is not orientable and has no boundary is non-orientable. Topologically, a hypermap is orientable if its underlying surface is orientable, has no boundary if its underlying surface has no boundary, and is bipartite if we can divide its set of vertices in two parts in such a way that consecutive vertices around an edge or a face are in alternate parts.

Henceforth, we use $|\mathcal{H}|$ to denote the number of flags of $\mathcal{H}$ instead of its set of flags, for simplicity. The numbers of vertices, edges and faces of $\mathcal{H}$ are denoted by $V(\mathcal{H}), E(\mathcal{H})$ and $F(\mathcal{H})$. The Euler characteristic of $\mathcal{H}$ is the integer

$$
\chi(\mathcal{H})=V(\mathcal{H})+E(\mathcal{H})+F(\mathcal{H})-\frac{|\mathcal{H}|}{2}
$$

and the genus of $\mathcal{H}, g(\mathcal{H})$, is $(2-\chi(\mathcal{H})) / 2$ or $2-\chi(\mathcal{H})$ depending on whether $\mathcal{H}$ is orientable or not.

The valency of a vertex, edge or face of a hypermap without boundary is half its number of flags. A hypermap $\mathcal{U}$ is uniform if there are positive integers $\ell, m$ and $n$ such that all vertices have valency $\ell$, all edges have valency $m$ and all faces have valency $n$. The triple $(\ell, m, n)$ is called the type of $\mathcal{U}$. Regular hypermaps are uniform but, in general, 
bipartite-regular hypermaps are not uniform. However, given a biparte-regular hypermap $\mathcal{B}$, there are positive integers $\ell_{1}, \ell_{2}, m$ and $n$ (not necessarily distinct) such that all edges have valency $m$, all faces have valency $n$ and consecutive vertices around an edge or a face have alternate valencies $\ell_{1}$ and $\ell_{2}$. The quadruple $\left(\ell_{1}, \ell_{2} ; m ; n\right)$ is a bipartite-type of $\mathcal{B}$ (another is $\left(\ell_{2}, \ell_{1} ; m ; n\right)$, if $\ell_{1} \neq \ell_{2}$ ).

For any $\sigma \in S_{\{0,1,2\}}$ we have an automorphism $\bar{\sigma}$ of $\Delta$ defined by $R_{i} \bar{\sigma}=R_{i \sigma}$, for $i \in\{0,1,2\}$. This automorphism induces an operation $\mathrm{D}_{\sigma}$ on hypermaps by assigning to $\mathcal{H}$ the hypermap $\mathrm{D}_{\sigma}(\mathcal{H})$ with hypermap subgroup $H \bar{\sigma}$, called $\sigma$-dual of $\mathcal{H}$.

Throughout the last century many authors contributed to the classification of regular and chiral maps on surfaces of low genus. More recently, Conder [5] obtained lists of regular and chiral maps and hypermaps whose Euler characteristic is negative and greater than or equal to -200, up to isomorphism and duality, using the "LowIndexNormalSubgroups" routine in MAGMA. Bipartite-regular hypermaps have been classified on the sphere [2], the projective plane, the torus and the double torus [8].

Let $\mathcal{H}^{+}$be the hypermap with hypermap subgroup $H^{+}:=H \cap \Delta^{+}$. Obviously, $\mathcal{H}^{+}$is isomorphic to $\mathcal{H}$ if $\mathcal{H}$ is orientable. When $\mathcal{H}$ is not orientable, $\mathcal{H}^{+}$is a covering of $\mathcal{H}$ with twice the number of flags usually called the orientable double cover of $\mathcal{H}$.

The covering core $\mathcal{H}_{\Delta}$ of $\mathcal{H}$ and the closure cover $\mathcal{H}^{\Delta}$ of $\mathcal{H}$ are the hypermaps whose hypermap subgroups are the core $H_{\Delta}$ of $H$ in $\Delta$ and the normal closure $H^{\Delta}$ of $H$ in $\Delta$, respectively. While the covering core of $\mathcal{H}$ is the smallest regular hypermap covering $\mathcal{H}$, the closure cover of $\mathcal{H}$ is the largest regular hypermap covered by $\mathcal{H}$.

When $\mathcal{H}$ is $\Theta$-conservative resp. $\Theta$-regular, $\mathcal{H}^{+}, \mathcal{H}^{\Delta}$ and $\mathcal{H}_{\Delta}$ are also $\Theta$-conservative resp. $\Theta$-regular. In fact, both $\mathcal{H}$ and $\mathcal{H}^{\Delta}$ are $\Theta$-conservative resp. $\Theta$-regular or neither is.

Lemma 1. For every hypermap $\mathcal{H},\left(\mathcal{H}^{+}\right)^{\Delta} \rightarrow\left(\mathcal{H}^{\Delta}\right)^{+}$and $\left(\mathcal{H}^{+}\right)_{\Delta} \cong\left(\mathcal{H}_{\Delta}\right)^{+}$.

More generally, we have the following coverings:

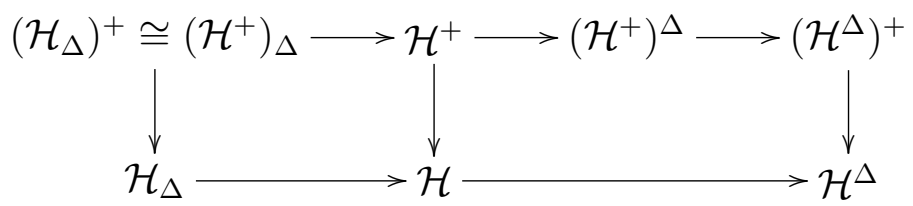

When $\mathcal{H}$ is $\Theta$-regular, for some normal subgroup $\Theta$ of index 2 in $\Delta$, but not orientable, $\left|\left(\mathcal{H}^{+}\right)^{\Delta}\right|$ is equal to $2\left|\left(\mathcal{H}^{\Delta}\right)^{+}\right|$or $\left|\left(\mathcal{H}^{\Delta}\right)^{+}\right|$depending on whether $\mathcal{H}_{\Delta}$ is orientable or not. Non-orientable bipartite-regular hypermaps whose covering core is orientable can be found on the projective plane (Chapter 3 of [8]) and on the Klein bottle (see Section 3).

\section{Constructions of bipartite hypermaps}

In this section we see some properties of the constructions of bipartite hypermaps described in $[2,8]$. These constructions are induced from group epimorphisms from $\Delta^{\hat{0}}$ to $\Delta$ and generalize the correspondence between hypermaps and bipartite maps presented 
by Walsh in [12]. Unless otherwise stated, we shall assume throughout this paper that $\varphi$ is an epimorphism from $\Delta^{\hat{0}}$ to $\Delta$.

Let $\mathcal{H}$ be a hypermap with hypermap subgroup $H$. For every conjugate $H^{g}$ of $H$ in $\Delta$, $H^{g} \varphi^{-1}$ is a conjugate of $H \varphi^{-1}$ in $\Delta^{\hat{0}}$ and hence in $\Delta$. In other words, $H^{g} \varphi^{-1}$ and $H \varphi^{-1}$ are hypermap subgroups for the same hypermap. Because of this, $\varphi$ induces an operator $\widetilde{\varphi}$ between the class of hypermaps and the class of bipartite hypermaps. In $[2,8]$, the hypermap $\widetilde{\varphi}(\mathcal{H})$ was denoted by $\mathcal{H}^{\varphi^{-1}}$. The following result lists some basic properties of these constructions of bipartite hypermaps.

Lemma 2. Let $\mathcal{H}$ and $\mathcal{G}$ be hypermaps and $\Theta$ a normal subgroup of $\Delta$. Then:

1. $\widetilde{\varphi}(\mathcal{H})$ has twice the number of flags of $\mathcal{H}$.

2. $\widetilde{\varphi}(\mathcal{H})$ is bipartite-regular if and only if $\mathcal{H}$ is regular. Moreover, $\widetilde{\varphi}(\mathcal{H})$ is $\Theta \varphi^{-1}$-regular if and only if $\mathcal{H}$ is $\Theta$-regular.

3. If $\mathcal{H}$ covers $\mathcal{G}$, then $\widetilde{\varphi}(\mathcal{H})$ covers $\widetilde{\varphi}(\mathcal{G})$.

4. If $\mathcal{H}$ is isomorphic to $\mathcal{G}$, then $\widetilde{\varphi}(\mathcal{H})$ is isomorphic to $\widetilde{\varphi}(\mathcal{G})$.

5. If $\widetilde{\varphi}(\mathcal{H})$ is regular, then $\mathcal{H}$ is regular.

More generally, if $\widetilde{\varphi}(\mathcal{H})$ is $\Theta$-regular, then $\mathcal{H}$ is $\left(\Theta \cap \Delta^{\hat{0}}\right) \varphi$-regular.

6. $\operatorname{Aut}(\mathcal{H})$ is either $\operatorname{Aut}(\widetilde{\varphi}(\mathcal{H}))$ or a subgroup of $\operatorname{Aut}(\widetilde{\varphi}(\mathcal{H}))$ of index 2 .

Proof. Let $H$ and $G$ be hypermap subgroups of $\mathcal{H}$ and $\mathcal{G}$.

1. Because $\varphi$ is an epimorphism, $\left(\Delta^{\hat{0}}: H \varphi^{-1}\right)=(\Delta: H)$ and hence

$$
|\widetilde{\varphi}(\mathcal{H})|=\left(\Delta: H \varphi^{-1}\right)=\left(\Delta: \Delta^{\hat{0}}\right)\left(\Delta^{\hat{0}}: H \varphi^{-1}\right)=2(\Delta: H)=2|\mathcal{H}|
$$

2. Since $\varphi$ is an epimorphism, $H \varphi^{-1} \triangleleft \Theta \varphi^{-1}$ if and only if $H \triangleleft \Theta$. 3. Let $H \subseteq G^{s}$ and $s=t \varphi$, for some $t \in \Delta^{\hat{0}}$, then $H \varphi^{-1} \subseteq G^{s} \varphi^{-1}=G^{t \varphi} \varphi^{-1}=\left(G \varphi^{-1}\right)^{t}$.

4. Follows from the proof of 3 by replacing " $\subseteq$ " with " $="$.

5. When $H \varphi^{-1}$ is normal in $\Theta$, it is also normal in $\Theta \cap \Delta^{\hat{0}}$, and so $H=H \varphi^{-1} \varphi$ is normal in $\left(\Theta \cap \Delta^{\hat{0}}\right) \varphi$.

6. Given $g \in \Delta^{\hat{0}}, H=H^{g \varphi} \Leftrightarrow H \varphi^{-1}=\left(H^{g \varphi}\right) \varphi^{-1} \Leftrightarrow H \varphi^{-1}=\left(H \varphi^{-1}\right)^{g}$ since $\varphi$ is an epimorphism and $\left(H^{g \varphi}\right) \varphi^{-1}=\left(H \varphi^{-1}\right)^{g}$ (see 1 of Lemma 8 of [2], for instance). Consequently, $\left(\mathrm{N}_{\Delta}(H)\right) \varphi^{-1}=\mathrm{N}_{\Delta}\left(H \varphi^{-1}\right) \cap \Delta^{\hat{0}}$, and so

$$
\operatorname{Aut}(\mathcal{H}) \cong \mathrm{N}_{\Delta}(H) / H \cong\left(N_{\Delta}(H)\right) \varphi^{-1} / H \varphi^{-1} \cong\left(\mathrm{N}_{\Delta}\left(H \varphi^{-1}\right) \cap \Delta^{\hat{0}}\right) / H \varphi^{-1}
$$

Because of this, $\operatorname{Aut}(\mathcal{H})$ is either $\operatorname{Aut}(\widetilde{\varphi}(\mathcal{H}))$ or a subgroup of $\operatorname{Aut}(\widetilde{\varphi}(\mathcal{H}))$ of index 2 depending on whether $\mathrm{N}_{\Delta}\left(H \varphi^{-1}\right)$ is contained in $\Delta^{\hat{0}}$ or not. 
The first 4 properties of Lemma 2 are just a reformulation of Lemma 1.6.1 of [8], 2 generalizes Theorems 10 and 12 of [2], and 5 generalizes Theorem 10 of [6].

When $\mathcal{B}$ is a bipartite hypermap such that $\mathcal{B} \cong \widetilde{\varphi}(\mathcal{K})$, for some hypermap $\mathcal{K}$, we say that $\mathcal{B}$ is constructed using $\varphi$. We denote the class of all bipartite hypermaps constructed using $\varphi$ by im $\widetilde{\varphi}$. The following result gives us a condition for seeing if a hypermap belongs to $\operatorname{im} \widetilde{\varphi}$ or not.

Lemma 3. Let $\mathcal{B}$ be a bipartite hypermap with hypermap subgroup $B$. Then $\mathcal{B} \in \operatorname{im} \widetilde{\varphi}$ if and only if $\operatorname{ker} \varphi \subseteq B^{g}$, for some $g \in \Delta$. Because $\operatorname{ker} \varphi$ is a normal subgroup of $\Delta^{0}$, $\mathcal{B} \in \operatorname{im} \widetilde{\varphi}$ if and only if $\operatorname{ker} \varphi \subseteq B$ or $(\operatorname{ker} \varphi)^{R_{0}} \subseteq B$.

Proof. If $\mathcal{B} \cong \widetilde{\varphi}(\mathcal{K})$, for some hypermap $\mathcal{K}$ with hypermap subgroup $K$, then there is $g \in \Delta$ such that $B^{g}=K \varphi^{-1}$ and hence $\operatorname{ker} \varphi=1 \varphi^{-1} \subseteq K \varphi^{-1}=B^{g}$.

Conversely, assume that $\operatorname{ker} \varphi \subseteq B^{g}$, for some $g \in \Delta$. Then $B^{g} \subseteq\left(\Delta^{0}\right)^{g}=\Delta^{\hat{0}}$, $B^{g}=B^{g} \cdot \operatorname{ker} \varphi=\left(B^{g}\right) \varphi \varphi^{-1}$ and so $\mathcal{B} \cong \widetilde{\varphi}(\mathcal{H})$, where $\mathcal{H}$ is the hypermap with hypermap subgroup $H:=\left(B^{g}\right) \varphi$.

Finally, we remark that $\mathcal{B} \in \operatorname{im} \widetilde{\varphi}$ is equivalent to $(\operatorname{ker} \varphi)^{g^{-1}} \subseteq B$ for some $g \in \Delta$, and since $\operatorname{ker} \varphi=1 \varphi^{-1}$ is a normal subgroup of $\Delta \varphi^{-1}=\Delta^{\hat{0}},(\operatorname{ker} \varphi)^{g^{-1}}$ is $\operatorname{ker} \varphi$ or $(\operatorname{ker} \varphi)^{R_{0}}$ depending on whether $g$ is in $\Delta^{\hat{0}}$ or not.

Theorem 4. Let $\mathcal{A}$ and $\mathcal{B}$ be bipartite hypermaps.

1. If $\mathcal{A} \rightarrow \mathcal{B}$ and $\mathcal{A} \in \operatorname{im} \tilde{\varphi}$, then $\mathcal{B} \in \operatorname{im} \tilde{\varphi}$.

2. If $\mathcal{B}^{+} \in \operatorname{im} \widetilde{\varphi}$, then $\mathcal{B} \in \operatorname{im} \widetilde{\varphi}$.

3. If $\mathcal{B}_{\Delta} \in \operatorname{im} \widetilde{\varphi}$, then $\mathcal{B} \in \operatorname{im} \widetilde{\varphi}$.

4. If $\mathcal{B} \in \operatorname{im} \widetilde{\varphi}$, then $\mathcal{B}^{\Delta} \in \operatorname{im} \widetilde{\varphi}$.

Proof. 1. Follows from Lemma 3 and the fact that if $A$ and $B$ are hypermap subgroups for $\mathcal{A}$ and $\mathcal{B}$, then $A \subseteq B^{h}$, for some $h \in \Delta$.

2. and 3. Follow from 1 because both $\mathcal{B}^{+}$and $\mathcal{B}_{\Delta}$ are bipartite and cover $\mathcal{B}$.

4. Follows from 1 since $\mathcal{B}^{\Delta}$ is a bipartite hypermap covered by $\mathcal{B}$.

Theorem 5. $\widetilde{\varphi}\left(\mathcal{H}_{\Delta}\right)$ is covered by $\widetilde{\varphi}(\mathcal{H})_{\Delta}$ and $\widetilde{\varphi}\left(\mathcal{H}^{\Delta}\right)$ covers $\widetilde{\varphi}(\mathcal{H})^{\Delta}$.

Proof. First of all note that $\widetilde{\varphi}(\mathcal{H})_{\Delta}$ and $\widetilde{\varphi}(\mathcal{H})^{\Delta}$ are both bipartite since $\widetilde{\varphi}(\mathcal{H})$ is bipartite. Because $\varphi$ is an epimorphism, $\left(H \varphi^{-1}\right)_{\Delta} \varphi$ and $\left(H \varphi^{-1}\right)^{\Delta} \varphi$ are normal subgroups of $\Delta$ such that $\left(H \varphi^{-1}\right)_{\Delta} \varphi \subseteq H \varphi^{-1} \varphi=H$ and $H=H \varphi^{-1} \varphi \subseteq\left(H \varphi^{-1}\right)^{\Delta} \varphi$. Hence, $\left(H \varphi^{-1}\right)_{\Delta} \varphi \subseteq H_{\Delta}$ and $H^{\Delta} \subseteq\left(H \varphi^{-1}\right)^{\Delta} \varphi$. Finally,

$$
\left(H \varphi^{-1}\right)_{\Delta} \subseteq\left(H \varphi^{-1}\right)_{\Delta} \operatorname{ker} \varphi=\left(H \varphi^{-1}\right)_{\Delta} \varphi \varphi^{-1} \subseteq H_{\Delta} \varphi^{-1}
$$

and

$$
H^{\Delta} \varphi^{-1} \subseteq\left(H \varphi^{-1}\right)^{\Delta} \varphi \varphi^{-1}=\left(H \varphi^{-1}\right)^{\Delta} \operatorname{ker} \varphi=\left(H \varphi^{-1}\right)^{\Delta}
$$

because $\operatorname{ker} \varphi=1 \varphi^{-1} \subseteq H \varphi^{-1} \subseteq\left(H \varphi^{-1}\right)^{\Delta}$. 
Hence, we have the following coverings:

$$
\widetilde{\varphi}(\mathcal{H})_{\Delta} \rightarrow \widetilde{\varphi}\left(\mathcal{H}_{\Delta}\right) \rightarrow \widetilde{\varphi}(\mathcal{H}) \rightarrow \widetilde{\varphi}\left(\mathcal{H}^{\Delta}\right) \rightarrow \widetilde{\varphi}(\mathcal{H})^{\Delta}
$$

In addition, $\widetilde{\varphi}(\mathcal{H})_{\Delta}$ is isomorphic to $\widetilde{\varphi}\left(\mathcal{H}_{\Delta}\right)$ if and only if $\widetilde{\varphi}\left(\mathcal{H}_{\Delta}\right)$ is regular, $\widetilde{\varphi}\left(\mathcal{H}_{\Delta}\right)$ and $\widetilde{\varphi}\left(\mathcal{H}^{\Delta}\right)$ are isomorphic to $\widetilde{\varphi}(\mathcal{H})$ if and only if $\mathcal{H}$ is regular and $\widetilde{\varphi}\left(\mathcal{H}^{\Delta}\right)$ is isomorphic to $\widetilde{\varphi}(\mathcal{H})^{\Delta}$ if and only if $\widetilde{\varphi}\left(\mathcal{H}^{\Delta}\right)$ is regular.

Now we prove a technical result.

Lemma 6. 1. If $g$ is a conjugate of $R_{1}, R_{2}, R_{1}{ }^{R_{0}}$ or $R_{2}{ }^{R_{0}}$ in $\Delta^{0}$, then $g \varphi$ is either 1 or a conjugate of $R_{0}, R_{1}$ or $R_{2}$ in $\Delta$. In addition, $g \in \Delta^{+} \varphi^{-1}$ if and only if $g \in \operatorname{ker} \varphi$.

2. If $h$ is a conjugate of $R_{0}, R_{1}$ or $R_{2}$ in $\Delta$, then there is a conjugate $g$ of $R_{1}, R_{2}$, $R_{1}{ }^{R_{0}}$ or $R_{2}{ }^{R_{0}}$ in $\Delta^{\hat{0}}$ such that $g \varphi=h$.

Proof. 1. Since $(g \varphi)^{2}=\left(g^{2}\right) \varphi=1 \varphi=1, g \varphi$ has order 1 or 2 . If $g \varphi$ has order $1, g \varphi=1$ and $g \in \operatorname{ker} \varphi$. When $g \varphi$ has order 2, the torsion theorem for free products (Theorem 1.6 in $\S I V .1$ of [10]) guarantees that $g \varphi$ is a conjugate of $R_{0}, R_{1}$ or $R_{2}$ in $\Delta$. Because no conjugate of $R_{0}, R_{1}$ or $R_{2}$ belongs to $\Delta^{+}$, the only element of finite order in $\Delta^{+}$is 1. Therefore, $g \varphi \in \Delta^{+}$if and only if $g \varphi=1$, or equivalently, $g \in \Delta^{+} \varphi^{-1}$ if and only if $g \in \operatorname{ker} \varphi$.

2. Let $S=\left\{R_{1}, R_{2}, R_{1}{ }^{R_{0}}, R_{2}{ }^{R_{0}}\right\},\{i, j, k\}=\{0,1,2\}$ and $h=R_{k}{ }^{c}$, for some $c \in \Delta$. The set $S \varphi$ has a conjugate of $R_{k}$ in $\Delta$. For otherwise all elements of $S \varphi$ would be either 1 or conjugates of $R_{i}$ or $R_{j}$ in $\Delta$, and so $\Delta^{\hat{0}} \varphi=\langle S\rangle \varphi=\langle S \varphi\rangle \subseteq\left\langle R_{i}, R_{j}\right\rangle^{\Delta}=\Delta^{\hat{k}} \neq \Delta$. Let $s \in S$ be such that $s \varphi=R_{k}^{b}$, for some $b \in \Delta$. Finally, let $a \in \Delta^{0}$ be such that $a \varphi=b^{-1} c$. Then $g:=s^{a}$ is a conjugate of $R_{1}, R_{2}, R_{1}{ }^{R_{0}}$ or $R_{2}{ }^{R_{0}}$ in $\Delta^{\hat{0}}$ such that $g \varphi=h$.

Theorem 7. 1. If $\widetilde{\varphi}(\mathcal{H})$ has no boundary, then $\mathcal{H}$ has no boundary.

2. If $\widetilde{\varphi}(\mathcal{H})$ is orientable, then $\mathcal{H}$ is orientable.

Proof. Let $H$ be a hypermap subgroup for $\mathcal{H}$.

1. If $\mathcal{H}$ has boundary, then there is a conjugate $h$ of $R_{0}, R_{1}$ and $R_{2}$ such that $h \in H$. By 2 . of Lemma 6, there is a conjugate $g$ of $R_{1}, R_{2}, R_{1}{ }^{R_{0}}$ or $R_{2}{ }^{R_{0}}$ in $\Delta^{\hat{0}}$ such that $g \varphi=h \in H$. It follows that $g \in H \varphi^{-1}$ and hence $\widetilde{\varphi}(\mathcal{H})$ has boundary.

2. Let $h=h_{1} \cdots h_{k} \in H$, where $h_{1}, \ldots, h_{k} \in\left\{R_{0}, R_{1}, R_{2}\right\}$. By 2. of Lemma 6 , there are conjugates $g_{1}, \ldots, g_{k}$ of $R_{1}, R_{2}, R_{1}{ }^{R_{0}}$ or $R_{2}{ }^{R_{0}}$ in $\Delta^{\hat{0}}$ such that $g_{i} \varphi=h_{i}$, for all $i \in\{1, \ldots, k\}$. Since $g=g_{1} \cdots g_{k} \in \Delta^{\hat{0}}$ and $g \varphi=h \in H, g \in H \varphi^{-1}$. In addition, $g \in \Delta^{+}$ if and only if $k$ is even, that is, if and only if $h \in \Delta^{+}$. Consequently, if $H \nsubseteq \Delta^{+}$, then $H \varphi^{-1} \nsubseteq \Delta^{+}$, that is, if $\mathcal{H}$ is not orientable, then $\widetilde{\varphi}(\mathcal{H})$ is not orientable.

We now come to the main result of this section.

Theorem 8. Let $\mathcal{H}, \mathcal{N}$ and $\mathcal{O}$ be hypermaps such that $\mathcal{N}$ has no boundary and $\mathcal{O}$ is orientable. The following conditions are equivalent:

1. $R_{1}, R_{2}, R_{1}^{R_{0}}, R_{2}{ }^{R_{0}} \notin \operatorname{ker} \varphi$. 
2. $R_{1}, R_{2}, R_{1}^{R_{0}}, R_{2}^{R_{0}} \notin \Delta^{+} \varphi^{-1}$.

3. $\Delta^{+} \varphi^{-1}=\Delta^{+} \cap \Delta^{\hat{0}}=\Delta^{+0 \hat{0}}$.

4. $\operatorname{ker} \varphi \subseteq \Delta^{+}$.

5. $\widetilde{\varphi}(\mathcal{N})$ has no boundary.

6. $\widetilde{\varphi}(\mathcal{O})$ is orientable.

7. $\widetilde{\varphi}\left(\mathcal{H}^{+}\right) \cong \widetilde{\varphi}(\mathcal{H})^{+}$.

8. $\widetilde{\varphi}(\mathcal{H})^{+} \in \operatorname{im} \widetilde{\varphi}$.

Proof. Let $H, N$ and $O$ be hypermap subgroups for $\mathcal{H}, \mathcal{N}$ and $\mathcal{O}$, respectively. $(1 \Leftrightarrow 2)$ By 1 . of Lemma 6 .

$(2 \Rightarrow 3)$ Let $S:=\left\{g \in \Delta^{\hat{0}} \mid g \in \Delta^{+} \Leftrightarrow g \in \Delta^{+} \varphi^{-1}\right\}$. Then:

- $R_{1}, R_{2}, R_{1}^{R_{0}}, R_{2}^{R_{0}} \in S \subseteq \Delta^{\hat{0}}$, and so $S \neq \varnothing$,

- for all $g, h \in S, g h \in S$, since

$$
\begin{aligned}
g h \in \Delta^{+} \varphi^{-1} & \Leftrightarrow(g h) \varphi=(g \varphi)(h \varphi) \in \Delta^{+} \\
& \Leftrightarrow g \varphi, h \varphi \in \Delta^{+} \text {or } g \varphi, h \varphi \notin \Delta^{+} \\
& \Leftrightarrow g, h \in \Delta^{+} \varphi^{-1} \text { or } g, h \notin \Delta^{+} \varphi^{-1} \\
& \Leftrightarrow g, h \in \Delta^{+} \text {or } g, h \notin \Delta^{+} \\
& \Leftrightarrow g h \in \Delta^{+},
\end{aligned}
$$

- for all $g \in S, g^{-1} \in S$, because

$$
g^{-1} \in \Delta^{+} \varphi^{-1} \Leftrightarrow g \in \Delta^{+} \varphi^{-1} \Leftrightarrow g \in \Delta^{+} \Leftrightarrow g^{-1} \in \Delta^{+} .
$$

It follows that $S$ is a subgroup of $\Delta^{\hat{0}}$ containing $\Delta^{\hat{0}}$, and so $S=\Delta^{\hat{0}}$. Therefore,

$$
g \in \Delta^{+} \Leftrightarrow g \varphi \in \Delta^{+} \Leftrightarrow g \in \Delta^{+} \varphi^{-1}
$$

for all $g \in \Delta^{\hat{0}}$, that is, $\Delta^{+} \cap \Delta^{\hat{0}}=\Delta^{+} \varphi^{-1} \cap \Delta^{\hat{0}}=\Delta^{+} \varphi^{-1}$.

$(3 \Rightarrow 6)$ Because $O \varphi^{-1} \subseteq \Delta^{\hat{0}}, O \subseteq \Delta^{+} \Leftrightarrow O \varphi^{-1} \subseteq \Delta^{+} \varphi^{-1}=\Delta^{+} \cap \Delta^{\hat{0}} \Leftrightarrow O \varphi^{-1} \subseteq \Delta^{+}$.

$(6 \Rightarrow 4) \operatorname{ker} \varphi=1 \varphi^{-1} \subseteq O \varphi^{-1} \subseteq \Delta^{+}$.

$(4 \Rightarrow 1)$ Because $R_{1}, R_{2}, R_{1} R_{0}, R_{2} R_{0} \notin \Delta^{+}$.

(2 $\Rightarrow 5$ ) Assume that $R_{1}, R_{2}, R_{1}{ }^{R_{0}}, R_{2}{ }^{R_{0}} \notin \Delta^{+} \varphi^{-1}$. Let $g$ be a conjugate of $R_{1}, R_{2}, R_{1} R_{0}$ or $R_{2}{ }^{R_{0}}$ in $\Delta^{\hat{0}}$. Since $\Delta^{+} \varphi^{-1}$ is a normal subgroup of $\Delta^{\hat{0}}, g \notin \Delta^{+} \varphi^{-1}$. In particular, $g \varphi \neq 1 \in \Delta^{+}$. By 1 . of Lemma $6, g \varphi$ is a conjugate of $R_{0}, R_{1}$ or $R_{2}$ in $\Delta$. Since $\mathcal{N}$ has no boundary, $g \varphi \notin N$, and so $g \notin N \varphi^{-1}$. Because of this, no conjugate of $R_{1}, R_{2}, R_{1} R_{0}$ or $R_{2}{ }^{R_{0}}$ in $\Delta^{\hat{0}}$ is in $N \varphi^{-1}$, that is, $\widetilde{\varphi}(\mathcal{N})$ has no boundary. 


\begin{tabular}{|l||l|l|l|l|l|}
\hline$g$ & $g \varphi_{1}$ & $g \varphi_{2}$ & $g \varphi_{3}$ & $g \varphi_{4}$ & $g \varphi_{5}$ \\
\hline \hline$R_{1}$ & $R_{1}$ & $R_{1}$ & $R_{1}$ & $R_{1}$ & $R_{1}$ \\
\hline$R_{2}$ & $R_{2}$ & $R_{2}$ & $R_{2}$ & $R_{2}$ & $R_{2}$ \\
\hline$R_{1}{ }^{R_{0}}$ & $R_{0}$ & $R_{0}$ & $R_{2}$ & $R_{0}$ & $R_{1}{ }^{R_{0}}$ \\
\hline$R_{2}{ }^{R_{0}}$ & $R_{2}$ & $R_{0}$ & $R_{0}$ & $R_{1}$ & $R_{0}$ \\
\hline
\end{tabular}

Table 1: The images of $R_{1}, R_{2}, R_{1}^{R_{0}}$ and $R_{2}{ }^{R_{0}}$ by $\varphi_{1}, \varphi_{2}, \varphi_{3}, \varphi_{4}$ and $\varphi_{5}$.

( $5 \Rightarrow 2$ ) Let $g$ be $R_{1}, R_{2}, R_{1}{ }^{R_{0}}$ or $R_{2}{ }^{R_{0}}$. Since $\widetilde{\varphi}(\mathcal{N})$ has no boundary, $g \notin N \varphi^{-1}$ and hence $g \varphi \neq 1 \in N$. By 1 . of Lemma $6, g \varphi$ is a conjugate of $R_{0}, R_{1}$ or $R_{2}$ in $\Delta$. In particular $g \notin \Delta^{+}$, or equivalently, $g \notin \Delta^{+} \varphi^{-1}$.

$(3 \Rightarrow 7)$ Since $\left(H^{+}\right) \varphi^{-1}=\left(H \cap \Delta^{+}\right) \varphi^{-1}$ and $\left(H \varphi^{-1}\right)^{+}=\left(H \varphi^{-1}\right) \cap \Delta^{+}$are hypermap subgroups for $\widetilde{\varphi}\left(\mathcal{H}^{+}\right)$and $\widetilde{\varphi}(\mathcal{H})^{+}$, and

$\left(H \cap \Delta^{+}\right) \varphi^{-1}=H \varphi^{-1} \cap \Delta^{+} \varphi^{-1}=H \varphi^{-1} \cap\left(\Delta^{+} \cap \Delta^{\hat{0}}\right)=\left(H \varphi^{-1} \cap \Delta^{\hat{0}}\right) \cap \Delta^{+}=\left(H \varphi^{-1}\right) \cap \Delta^{+}$, $\widetilde{\varphi}\left(\mathcal{H}^{+}\right)$and $\widetilde{\varphi}(\mathcal{H})^{+}$are isomorphic.

$(7 \Rightarrow 8)$ Clearly, $\widetilde{\varphi}\left(\mathcal{H}^{+}\right) \in \operatorname{im} \widetilde{\varphi}$.

$(8 \Rightarrow 4)$ By Lemma 3 and because $\left(H \varphi^{-1}\right)^{+}$is a hypermap subgroup for $\widetilde{\varphi}(\mathcal{H})^{+}$, we have $\operatorname{ker} \varphi \subseteq\left(\left(H \varphi^{-1}\right)^{+}\right)^{g}=\left(H \varphi^{-1} \cap \Delta^{+}\right)^{g} \subseteq\left(\Delta^{+}\right)^{g}=\Delta^{+}$, for some $g \in \Delta$.

As an immediate corollary to Theorem 8 (and Theorems 4 and 7) we get the following.

Corollary 9. Let $\varphi$ be an epimorphism such that $R_{1}, R_{2}, R_{1}{ }^{R_{0}}, R_{2}{ }^{R_{0}} \notin \operatorname{ker} \varphi$. Then:

1. Both $\mathcal{H}$ and $\widetilde{\varphi}(\mathcal{H})$ are orientable or neither is.

2. Both $\mathcal{H}$ and $\widetilde{\varphi}(\mathcal{H})$ have boundary or neither has.

3. If $\mathcal{B}$ is bipartite, then both $\mathcal{B}$ and $\mathcal{B}^{+}$belong to im $\widetilde{\varphi}$ or neither does.

Corollary 10. If $\mathcal{B} \cong \widetilde{\varphi}(\mathcal{H})$ is a non-orientable hypermap, then $\mathcal{B}^{+} \cong \widetilde{\varphi}\left(\mathcal{H}^{+}\right)$.

Proof. By Theorem 7, $\mathcal{H}$ has no boundary. The proof now follows from Theorem 8.

We now give several examples of epimorphisms from $\Delta^{\hat{0}}$ to $\Delta$.

As noted in [2], the Reidemeister-Schreier rewriting process [9] can be used to show that

$$
\Delta^{\hat{0}}=\left\langle R_{1}\right\rangle *\left\langle R_{2}\right\rangle *\left\langle R_{1}^{R_{0}}\right\rangle *\left\langle R_{2}^{R_{0}}\right\rangle .
$$

Because of this, every epimorphism from $\Delta^{\hat{0}}$ to $\Delta$ is completely determined by the images of $R_{1}, R_{2}, R_{1}{ }^{R_{0}}$ and $R_{2}{ }^{R_{0}}$. Let $\varphi_{1}, \varphi_{2}, \varphi_{3}, \varphi_{4}, \varphi_{5}$ be the epimorphisms from $\Delta^{\hat{0}}$ to $\Delta$ whose images of $R_{1}, R_{2}, R_{1}{ }^{R_{0}}$ and $R_{2}{ }^{R_{0}}$ are listed in Table 1 . The construction induced by $\varphi_{1}$ is the well known Walsh's correspondence between hypermaps and bipartite maps [12], and $\varphi_{2}$ induces a construction described in $[2,8]$. Note that $\varphi_{4}=(12) \varphi_{3}(12)$.

Let $\varphi_{i}$ be one of these epimorphisms. Since $R_{1}, R_{2}, R_{1}{ }^{R_{0}}, R_{2}{ }^{R_{0}} \notin \operatorname{ker} \varphi_{i}$, the construction induced by $\varphi_{i}$ has the following properties: 
- both $\mathcal{H}$ and $\widetilde{\varphi}_{i}(\mathcal{H})$ are orientable or neither is,

- both $\mathcal{H}$ and $\widetilde{\varphi}_{i}(\mathcal{H})$ have boundary or neither has,

- $\widetilde{\varphi}_{i}\left(\mathcal{H}^{+}\right) \cong \widetilde{\varphi}_{i}(\mathcal{H})^{+}$,

- if $\mathcal{B}$ is bipartite, then both $\mathcal{B}$ and $\mathcal{B}^{+}$are constructed using $\varphi_{i}$ or neither is.

Although $\mathcal{H}, \widetilde{\varphi}_{1}(\mathcal{H})$ and $\widetilde{\varphi}_{2}(\mathcal{H})$ all have the same underlying surface [2], in general $\mathcal{H}$ and $\widetilde{\varphi}(\mathcal{H})$ may not have the same Euler characteristic.

Lemma 11. Let $\mathcal{R}$ be a regular hypermap of type $(\ell, m, n)$. Then

1. $\widetilde{\varphi}_{1}(\mathcal{R})$ has bipartite-type $(\ell, m ; 2 ; 2 n)$ and $\chi\left(\widetilde{\varphi}_{1}(\mathcal{R})\right)=\chi(\mathcal{R})$;

2. $\widetilde{\varphi}_{2}(\mathcal{R})$ has bipartite-type $(1, \ell ; 2 m ; 2 n)$ and $\chi\left(\widetilde{\varphi}_{2}(\mathcal{R})\right)=\chi(\mathcal{R})$;

3. $\widetilde{\varphi}_{3}(\mathcal{R})$ has bipartite-type $(\ell, m ; 2 \ell ; 2 m)$ and $\chi\left(\widetilde{\varphi}_{3}(\mathcal{R})\right)=2(\chi(\mathcal{R})-F(\mathcal{R}))$;

4. $\widetilde{\varphi}_{4}(\mathcal{R})$ has bipartite-type $(\ell, n ; 2 \ell ; 2 n)$ and $\chi\left(\widetilde{\varphi}_{4}(\mathcal{R})\right)=2(\chi(\mathcal{R})-E(\mathcal{R}))$;

5. $\widetilde{\varphi}_{5}(\mathcal{R})$ has bipartite-type $(\ell, n ; 2 m ; n)$ and $\chi\left(\widetilde{\varphi}_{5}(\mathcal{R})\right)=\chi(\mathcal{R})+2 F(\mathcal{R})-\frac{|\mathcal{R}|}{2}$ if $n$ is even, or bipartite-type $(\ell, n ; 2 m ; 2 n)$ and $\chi\left(\widetilde{\varphi}_{5}(\mathcal{R})\right)=\chi(\mathcal{R})+F(\mathcal{R})-\frac{|\mathcal{R}|}{2}$ if $n$ is odd.

Proof. All proofs are similar, so we will only show 5 . Let $R$ be a hypermap subgroup for $\mathcal{R}$. Because the valencies of the edges and the faces of $\widetilde{\varphi}_{5}(\mathcal{R})$ are even, we may assume that $\widetilde{\varphi}_{5}(\mathcal{R})$ has bipartite-type $\left(p_{1}, p_{2} ; 2 q ; 2 r\right)$. Then $u_{1}=\ell, u_{2}=m$ and $u_{3}=n$ are the smallest positive integers such that $\left(R_{1} R_{2}\right)^{u_{1}},\left(R_{2} R_{0}\right)^{u_{2}},\left(R_{0} R_{1}\right)^{u_{3}} \in R$. Similarly, $v_{1}=p_{1}, v_{2}=p_{2}, v_{3}=q$ and $v_{4}=r$ are the smallest positive integers such that $\left(R_{1} R_{2}\right)^{v_{1}},\left(R_{1}{ }^{R_{0}} R_{2}{ }^{R_{0}}\right)^{v_{2}},\left(R_{2} R_{2}{ }^{R_{0}}\right)^{v_{3}},\left(R_{1}{ }^{R_{0}} R_{1}\right)^{v_{4}} \in R \varphi_{5}{ }^{-1}$. Since

$$
\begin{gathered}
\left(R_{1} R_{2}\right) \varphi_{5}=R_{1} R_{2}, \quad\left(R_{1}{ }^{R_{0}} R_{2}{ }^{R_{0}}\right) \varphi_{5}=R_{0} R_{1}, \\
\left(R_{2} R_{2}{ }^{R_{0}}\right) \varphi_{5}=\left(R_{1} R_{2}\right)^{-1} \quad \text { and } \quad\left(R_{1}{ }^{R_{0}} R_{1}\right) \varphi_{5}=\left(R_{0} R_{1}\right)^{-2},
\end{gathered}
$$

we have $p_{1}=\ell, p_{2}=n, q=\ell$, and $r=\frac{n}{\operatorname{gcd}(n, 2)}$, so $r$ is either $\frac{n}{2}$ or $n$, depending on whether $n$ is even or odd. The formula for the characteristic follows from Lemma 19 of [2].

Note that 1. and 2. of Lemma 11 are consequences of Theorems 10 and 12 of [2].

Two interesting questions which naturally occur are whether all bipartite-regular hypermaps are obtained from regular hypermaps using these constructions, and whether there is a surface (without boundary) having no bipartite-regular hypermaps. The answer to both questions is "no" as we will see in Sections 3 and 4. 


\section{Some bipartite hypermaps}

In this section we present bipartite hypermaps which are not obtained by the constructions described above.

Let $\mathcal{K}$ be the bipartite-regular hypermap with hypermap subgroup

$$
K:=\left\langle\left\{(u v)^{2} \mid u, v \in\left\{R_{1}, R_{2}, R_{1}{ }^{R_{0}}, R_{2}{ }^{R_{0}}\right\}\right\} \cup\left\{R_{2} R_{2}{ }^{R_{0}} R_{1}{ }^{R_{0}}\right\}\right\rangle^{\Delta^{\hat{0}}},
$$

One can see that $\mathcal{K}$ is a uniform hypermap of type $(2,4,4)$ on the Klein bottle with 16 flags. Its orientable double cover $\mathcal{T}:=\mathcal{K}^{+}$is also bipartite-regular and uniform of type $(2,4,4)$, but is on the torus and has 32 flags. A hypermap subgroup for $\mathcal{T}$ is

$$
T:=K \cap \Delta^{+}=\left\langle\left\{(u v)^{2} \mid u, v \in\left\{R_{1}, R_{2}, R_{1}{ }^{R_{0}}, R_{2}{ }^{R_{0}}\right\}\right\}\right\rangle^{\Delta^{\hat{0}}} .
$$

In fact, $\mathcal{T}$ is regular and the dual $\mathrm{D}_{(01)}(\mathcal{T})$ is the regular map denoted by $\{4,4\}_{2,0}$ in [7], by $\{i\}_{2}$ in [11], and by $(4,2,4)_{\left(\begin{array}{ll}2 & 0 \\ 0 & 2\end{array}\right)}$ in [8]. Since $\mathcal{K}$ is not regular, $\mathcal{T}$ is the covering core of $\mathcal{K}$, and hence $\left(\mathcal{K}^{+}\right)^{\Delta}$ and $\left(\mathcal{K}^{\Delta}\right)^{+}$are non-isomorphic.

When $\mathcal{B}$ is a bipartite-regular hypermap such that $\mathcal{B} \in \operatorname{im} \widetilde{\varphi}$ and $B$ is a hypermap subgroup for $\mathcal{B}$, then $\Delta^{\hat{0}} / B \cong \Delta / B \varphi$, and so $\Delta^{\hat{0}} / B$ is generated by up to 3 elements. Since no 3 elements generate $\Delta^{\hat{0}} / T \cong C_{2} \times C_{2} \times C_{2} \times C_{2}$, the hypermap $\mathcal{T}$ cannot be constructed using an epimorphism $\varphi$. Corollary 10 ensures that neither $\mathcal{K}$ can be constructed using $\varphi$. It also follows that $\Delta^{\hat{0}} / B$ being generated by up to 3 elements is a necessary but not sufficient condition for $\mathcal{B}$ being constructed using an epimorphism.

Let $\mathcal{P}_{2}$ be the unique regular hypermap on the sphere of type $(2,2,2)$ whose hypermap subgroup is $\Delta^{\prime}=\left\langle\left(R_{1} R_{2}\right)^{2},\left(R_{2} R_{0}\right)^{2},\left(R_{0} R_{1}\right)^{2}\right\rangle^{\Delta}$, the derived subgroup of $\Delta$. Then $\widetilde{\varphi}_{2}\left(\mathcal{P}_{2}\right)$ is a bipartite-regular hypermap of bipartite-type $(1,2 ; 4 ; 4)$ and its covering core $\widetilde{\varphi}_{2}\left(\mathcal{P}_{2}\right)_{\Delta}$ is $\mathcal{T}$ (see $[2,8]$ ), showing that the converse of 3 of Theorem 4 is not true.

\section{Surfaces with bipartite-regular hypermaps}

According to Theorems 10 and 12 of [2], the constructions induced by $\varphi_{1}$ and $\varphi_{2}$ guarantee the existence of bipartite-regular hypermaps on every surface which supports regular hypermaps. However, there are non-orientable surfaces having no regular hypermaps, such as the non-orientable surfaces of characteristic $0,-1,-16,-22,-25,-37$ and -46

(see $[4,13])$. Do these surfaces have bipartite-regular hypermaps? The answer is given by the following result.

Theorem 12. All surfaces have bipartite-regular hypermaps which are not regular and have vertices of valency 2.

Proof. Let $\mathcal{C}$ and $\mathcal{P C}$ denote the cube and the hemicube. Then $\widetilde{\varphi}_{1}(\mathcal{C})$ and $\widetilde{\varphi}_{1}(\mathcal{P C})$ are bipartite-regular hypermaps of bipartite-type $(2,3 ; 2 ; 4)$, the first on the sphere and the second on the projective plane. 
The existence of the required bipartite-regular hypermaps on every orientable surface of genus $g \geqslant 1$ follows from Theorem 23 of [2].

Finally, let $\mathcal{P} \mathcal{P}_{2 k}$ be the regular hypermap on the projective plane of type $(2,2,2 k)$, with $k$ vertices, $k$ edges, 1 face and $4 k$ flags. By Theorem 8 , the bipartite-regular hypermaps $\widetilde{\varphi}_{4}\left(\mathcal{P P}_{2 k}\right)$ and $\widetilde{\varphi}_{5}\left(\mathcal{P} \mathcal{P}_{2 k}\right)$ are both non-orientable. By Lemma 11

$$
\chi\left(\widetilde{\varphi}_{4}\left(\mathcal{P} \mathcal{P}_{2 k}\right)\right)=2-2 k \quad \text { and } \quad \chi\left(\widetilde{\varphi}_{5}\left(\mathcal{P} \mathcal{P}_{2 k}\right)\right)=2-(2 k-1),
$$

so the hypermaps $\widetilde{\varphi}_{4}\left(\mathcal{P} \mathcal{P}_{2 k}\right)$ and $\widetilde{\varphi}_{5}\left(\mathcal{P} \mathcal{P}_{2 k}\right)$ have genus $2 k$ and $2 k-1$, respectively.

According to Lemma 19 and Theorems 10 and 12 of [2], if $\mathcal{B}$ is a bipartite-regular hypermap with Euler characteristic 0, then either $\mathcal{B}$ is uniform of type $(2,4,4)$ or there is a regular hypermap $\mathcal{R}$ on the same surface as $\mathcal{B}$ such that $\mathcal{B}$ is isomorphic to $\widetilde{\varphi}_{1}(\mathcal{R})$ or $\widetilde{\varphi}_{2}(\mathcal{R})$ or $\mathrm{D}_{(12)}\left(\widetilde{\varphi}_{2}(\mathcal{R})\right)$. Since there are no regular hypermaps on the Klein bottle, all bipartite-regular hypermaps on the Klein bottle are uniform of type $(2,4,4)$. In other words, all vertices of a bipartite-regular on the Klein bottle have valency 2. Information on the bipartite-regular hypermaps on the torus can be found in Chapter 4 of [8].

\section{Acknowledgements}

The author would like to thank the anonymous referee for his/her valuable comments and suggestions.

This work was partially supported by FEDER funds through COMPETE-Operational Programme Factors of Competitiveness ("Programa Operacional Factores de Competitividade") and by Portuguese funds through the Center for Research and Development in Mathematics and Applications (University of Aveiro) and the Portuguese Foundation for Science and Technology ("FCT-Fundação para a Ciência e a Tecnologia"), within project PEst-C/MAT/UI4106/2011 with COMPETE number FCOMP-01-0124-FEDER-022690.

\section{References}

[1] Antonio Breda d'Azevedo, A theory of restricted regularity of hypermaps, J. Korean Math. Soc. 43 (2006), no. 5, 991-1018.

[2] Antonio Breda d'Azevedo and Rui Duarte, Bipartite-uniform hypermaps on the sphere, Electron. J. Combin. 14 (2007), 1-20.

[3] Antonio J. Breda d'Azevedo and Gareth A. Jones, Double coverings and reflexive abelian hypermaps, Beitr. Algebra Geom. 41 (2000), no. 2, 371-389.

[4] _ Rotary hypermaps of genus 2, Beitr. Algebra Geom. 42 (2001), no. 1, 39-58.

[5] Marston Conder, Marston Conder's home page, http://www . math . auckland.ac.nz/ conder/.

[6] David Corn and David Singerman, Regular hypermaps, European J. Combin. 9 (1988), no. 4, 337-351. 
[7] H.S.M. Coxeter and W.O.J. Moser, Generators and relations for discrete groups, 4th ed., Ergebnisse der Mathematik und ihrer Grenzgebiete, Bd. 14, Springer-Verlag, Berlin-Heidelberg-New York, 1980.

[8] Rui Duarte, 2-restrictedly-regular hypermaps of small genus, Ph.D. thesis, University of Aveiro, 2007.

[9] D. L. Johnson, Topics in the theory of groups presentations, London Math. Soc. Lecture Note Series, vol. 42, Cambridge University Press, 1980.

[10] R. C. Lyndon and P. E. Schupp, Combinatorial group theory, Springer-Verlag, BerlinHeidelberg-New York, 1977.

[11] David Singerman and Robert I. Syddall, Geometric structures on toroidal maps and elliptic curves, Math. Slovaca 50 (2000), no. 5, 495-512.

[12] T.R.S. Walsh, Hypermaps versus bipartite maps, J. Comb. Theory, Ser. B 18 (1975), $155-163$.

[13] Steve Wilson and Antonio Breda d'Azevedo, Surfaces having no regular hypermaps, Discrete Math. 277 (2004), no. 1-3, 241-274. 